\title{
L'importance de la culture française dans la vie culturelle et intellectuelle suédoise entre 1946 et 1995. Le cas du quotidien Svenska Dagbladet et la section Under strecket
}

Hans-Roland Johnsson

Université de Stockholm

Lors de la conférence Médiations interculturelles, trajectoires et circulations entre la France et la Suède de I945 à nos jours, qui s'est déroulée à Stockholm en janvier 20I4, maintes contributions ont démontré la diversité et la richesse de l'échange culturel entre la France et la Suède dans des domaines différents. Bien qu'il soit avéré que l'influence française sur la culture suédoise a joué un certain rôle, il est beaucoup plus difficile de la mesurer. Dans cet article, nous allons essayer de montrer que les articles sur la France et la culture française publiés dans la section Under strecket du quotidien Svenska Dagbladet peuvent nous servir de bon indicateur de l'impact de la culture française en Suède pendant les cinquante années après la deuxième guerre mondiale.

\section{Les échanges culturels dans un monde dynamique}

La culture d'un pays est rarement formée sans influences extérieures. Dans le monde moderne, les relations culturelles entre les pays sont complexes et se jouent normalement dans les deux directions simultanément, mais pas toujours dans les mêmes domaines ni au même de-

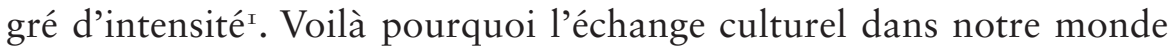
dynamique est un phénomène polyvalent. Il est pourtant difficile d'estimer l'importance de cet échange, puisqu'il s'agit souvent d'objets immatériels et plus abstraits que des marchandises qui sont enregistrées quand elles passent par la douane ${ }^{2}$.

How to cite this book chapter:

Johnsson, H.-R. 20I5. L'importance de la culture française dans la vie culturelle et intellectuelle suédoise entre I946 et 1995. Le cas du quotidien Svenska Dagbladet et la section Under strecket. In: Cedergren, M. et Briens, S. (eds.) Médiations interculturelles entre la France et la Suède. Trajectoires et circulations de 1945 à nos jours. Pp. 59-78. Stockholm: Stockholm University Press. DOI: http://dx.doi.org/Io.I6993/ bad.f. License: CC-BY 
L'importation d'une notion philosophique, d'une œuvre littéraire, cinématique ou musicale, se fait aussi par un canal, un contact intermédiaire, et un importateur - mais on parle alors plutôt de passeur au lieu d'importateur ${ }^{3}$, et de transfert plutôt que d'importation ${ }^{4}$. Ce passeur est aussi un observateur - il, ou elle, constate un phénomène (un livre, un auteur, un film, une notion philosophique etc.) sur la scène culturelle d'un autre pays et souhaite le faire connaître aussi au public de son propre pays.

\section{L'intérêt pour la culture française en Suède}

Au dix-huitième siècle, beaucoup de pays européens subirent l'influence de la culture française, ce qui eut des répercussions dans la société suédoise ${ }^{5}$. Même si l'enthousiasme pour tout ce qui était français diminua au cours du dix-neuvième siècle, la culture française fut toujours un point de référence important dans la culture suédoise ${ }^{6}$. Nous savons également qu'elle continua, d'une manière ou d'une autre, aussi bien avant qu'après la Seconde guerre mondiale, à jouer un rôle non-négligeable en Suède. Après 1945, elle pénétra par des voies multiples (des livres surtout des traductions de livres de fiction - des articles dans des revues spécialisées ou dans les quotidiens ${ }^{7}$, le cinéma, la télé, la radio, le tourisme ou les échanges commerciaux $\left.{ }^{8}\right)$.

\section{Comment mesurer l'importance de la culture française en Suède?}

Quel a alors été l'impact global de la culture française dans la vie culturelle et intellectuelle en Suède entre I946 et I995, ou, plus précisément, quel a été l'intérêt suédois pour la culture française durant cette période ? Cette présence française dans la vie culturelle suédoise après la guerre est indéniable et facile à constater. Pourtant, dire que la culture française dans tel ou tel contexte a joué un rôle important ne signifie pas a priori qu'elle a joué un très grand rôle.

Globalement, quand on regarde de près l'offre culturelle dans un pays comme la Suède et quand on essaie d'en démêler les influences diverses (dans une perspective synchronique et diachronique), on obtient plutôt un mélange dont la composante française - bien qu'importante - est une composante parmi d'autres tout aussi ou encore plus importantes 9 . Comment peut-on alors soutenir l'hypothèse que la culture française a été une référence importante dans la vie culturelle et intellectuelle en 
Suède ? Étant donné la diversité de la culture suédoise et son ouverture vers des influences venues de l'étranger, il n'est pas facile de déterminer à quel degré la culture française y a joué un rôle.

Pourtant, nous allons ici essayer de mesurer à quel point la culture française s'est imposée dans la culture suédoise après la guerre. Mesurer signifie donner des résultats chiffrés. Mesurer signifie aussi avoir un objet à mesurer. Cet objet doit, selon nous, remplir cinq critères :

(i) D'abord, il faut qu'il représente la culture dans son sens le plus large, c'est-à-dire qu'il dépasse l'art, la littérature et d'autres manifestations culturelles pour englober les multiples facettes de la société. Cela signifie que l'objet étudié reflète aussi la politique, la discussion intellectuelle globale, la science, la religion, la philosophie et ainsi de suite.

(ii) Il faut que l'objet soit stable/identique sur le plan temporel, c'est-à-dire il faut que ce soit le même objet qu'on étudie au début et à la fin de la période choisie.

(iii) L'objet étudié doit être reconnu comme un vecteur culturel par le grand public intellectuel.

(iv) L'objet doit refléter des domaines divers, selon (i.) ci-dessus, dans une perspective synchronique et diachronique.

(v) Il faut aussi, pour terminer, que la période sur laquelle l'objet est étudié soit suffisamment longue pour (a) pouvoir inclure beaucoup d'observations, et pour (b) écarter le risque d'étudier un phénomène limité dans le temps.

Si l'on accepte ces critères, nous supposons que l'objet le plus propice à étudier est un grand quotidien dont la diffusion couvre la Suède entière. Des journaux d'un contenu plus général n'ont guère existé en Suède. Cependant, il est difficile, pour ne pas dire impossible, de bien prendre en compte, dans la perspective qui est ici la nôtre, des articles dans toutes les sections d'un quotidien, surtout quand la longueur des articles est différente et le nombre d'articles publiés chaque jour change d'un mois à un autre ou d'une année à une autre - l'objet étudié n'est alors pas stable mais plutôt flou et difficile à saisir dans sa totalité.

\section{Svenska Dagbladet et la section Under strecket}

Heureusement, il y a, selon nous, un objet qui répond à nos demandes. Il existe une sorte particulière d'articles que l'on retrouve dans le 
quotidien Svenska Dagbladet et qui s'appelle Under strecket ("Sous la ligne $»)$.

Il s'agit d'un lieu unique dans la presse suédoise. C'est une partie d'une page du quotidien où se trouve un article ou, dans certains cas, un essai sur n'importe quel sujet culturel ou intellectuel, le plus souvent à l'occasion de la parution d'un livre dont la critique constitue l'article. Parmi les sujets abordés, on peut mentionner la littérature, l'art, le théâtre, la musique, l'architecture, l'histoire, la religion, la philosophie, la politique, le droit, l'économie, l'enseignement, la sociologie, la linguistique, les sciences naturelles, l'astronomie, la technique, l'informatique, la psychologie, la médecine, la santé, l'urbanisme, l'environnement, la nature, la géographie, et l'agriculture - tous présentés sous une forme destinée aux non-spécialistes mais tous basés sur une approche intellectuelle.

Ces catégories peuvent être sous-divisées (a) en articles dont le sujet est attaché à un pays autre que la Suède ; (b) en articles qui portent sur des sujets suédois ; (c) en articles qui traitent des sujets généraux sans que l'on puisse les attacher directement à un pays particulier. Le groupe (b) est le plus fréquent dans Under strecket.

C'est également l'article le plus long dans le quotidien. Le nombre de signes (espaces inclues) est normalement de I I 500 à I 2000 environ $^{\mathrm{IO}}$. Il est toujours placé en bas de page. Dans notre corpus, il se retrouve dans la section culturelle (au-dessous des critiques) à partir de 1965. Il est graphiquement séparé des autres articles par une ligne transversale ou par d'autres moyens graphiques (voilà pourquoi cet endroit s'appelle "Sous la ligne »), donc il est facilement repérable ${ }^{\mathrm{II}}$.

L'article est souvent écrit par des personnalités connues dans la vie intellectuelle en Suède. Autre caractéristique qui le distingue des autres articles est le fait qu'il est accompagné d'une reproduction de la signature de son auteur.

Un article Under strecket est publié chaque jour où sort le Svenska Dagbladet (donc environ 353 articles Under strecket par an).

Nous supposons que le choix fait par la rédaction reflète la demande du lectorat. Le lecteur du Svenska Dagbladet représente alors approximativement le Suédois qui s'intéresse aux questions culturelles et intellectuelles ${ }^{\mathrm{I}}$, Under strecket fonctionnant ainsi comme une sorte de baromètre de l'intérêt des intellectuels suédois pour la vie culturelle et intellectuelle en Suède ou dans d'autres pays ${ }^{\mathrm{I3}}$. 


\section{Les articles Under strecket qui portent sur la France}

Si Under strecket réunit tous les aspects qui ont trait à la vie culturelle et intellectuelle, le nombre d'articles sur la France à cet endroit dans Svenska Dagbladet devrait être un bon indicateur de l'impact de la culture française en Suède ou, en d'autres mots, de l'intérêt apporté à la culture française par le grand public intellectuel.

Nous n'avons pas lieu de croire que Svenska Dagbladet entre I946 et I995 s'adresse à un public particulièrement francophone. Quotidien conservateur modéré, la culture française ne devait pas a priori peser davantage que la culture anglaise, allemande ou nordique.

Le nombre d'articles sur la France - sur un total d'environ I7 640 articles publiés Under strecket entre I 946 et I 995 - constitue notre corpus ${ }^{\mathrm{I}}$.

Dans ce qui suit, nous définissons un article comme un article sur la France s'il porte explicitement sur la France $^{\text {Is }}$, par exemple s'il y est question d'un auteur/artiste français dont la production littéraire/artistique est à considérer comme une expression de la culture française ou si la production est ancrée dans la culture française ${ }^{16}$.

Il arrive assez souvent qu'un article ne porte qu' "à moitié » sur un sujet français - par exemple si l'article traite de la relation entre Strindberg et Zola ; dans un tel cas, l'article n'est compté que comme un « demi-article » (valeur « 0,5 » et non valeur «I $»)^{17}$. Un article dans lequel il est question d'un livre écrit par un Français sur un sujet général sans connexion directe avec la France ne fait pas partie de notre corpus. Dans certains cas, l'établissement du corpus entraîne des questions épineuses, mais dans l'ensemble nous estimons que le tri a été fait d'une façon conséquente.

Étant donné la grande diversité de sujets possibles dans un article publié Under strecket, sur quelle échelle évaluer la fréquence des articles sur la France ? Elle peut être mesurée d'une façon objective, chiffrée, mais il n'y a pas de critère fixe à l'aide duquel on peut objectivement l'évaluer. Il est évident, pour commencer, qu'un article chaque semaine est un chiffre impossible à atteindre - la concurrence des autres sujets est trop rude. Un article tous les quinze jours serait dans le contexte de Under strecket un excellent résultat. Un article par mois, par contre, serait un chiffre trop faible. Dans ce cas, on dira que l'intérêt pour la France est faible auprès des intellectuels suédois.

De fait, le chiffre pour la période I946-1995 est de I,9 article mensuel en moyenne - donc, presque un article sur la France tous les quinze jours - ce qui équivaut à 22,6 articles par an ou, pour la période dans 
sa totalité, plus de I I 30 articles. On peut alors - avec toute la prudence nécessaire - tirer la conclusion que l'intérêt chez les lecteurs intellectuels en Suède pour la France après la deuxième guerre mondiale a été élevé. Nous dirons même que la quantité d'articles publiés est étonnante vu le grand nombre de sujets que cette rubrique Under strecket peut embrasser.

Dans le graphique ${ }^{\circ} \mathrm{I}$, on voit que le nombre d'articles publiés est un peu moins élevé vers le début et vers la fin de la période. Pourtant, il n'y a pas de tendance claire sur la période prise dans sa totalité ${ }^{18}$; l'intérêt semble être relativement stable avec des chiffres fluctuant autour de 22 articles par an ${ }^{19}$.

\section{Les articles et leur contenu}

Si l'on regarde les sujets rangés dans le tableau $n^{\circ} \mathrm{I}^{20}$, on peut constater que les articles sur la littérature dominent : 44 pour cent des articles ont un sujet littéraire, bien que le nombre soit plus élevé pendant les premières que les dernières vingt-cinq années. Cette diminution est due à un chiffre moins important de contributions sur la littérature contemporaine ${ }^{21}$, tandis que les articles qui portent sur la littérature non-contemporaine deviennent plus fréquents ${ }^{22}$. À partir de I96I, Svenska Dagbladet publie presque chaque année deux articles Under strecket sur la saison théâtrale à Paris, signés Kjell Strömberg. Après sa mort, en I975, le nombre d'articles consacrés aux scènes parisiennes diminue sensiblement. ${ }^{23}$

L'augmentation du nombre d'articles dans le groupe « D'autres manifestations culturelles » est principalement due à un accroissement d'articles consacrés à la peinture française. L'augmentation des articles sous la rubrique " Histoire » est en partie imputable à un intérêt accru pour la Révolution française, occasionné par le bicentenaire en I989.

\section{Personnes mentionnées dans les articles}

Notre étude n'est pas pertinente au niveau des individus ${ }^{24}$. Pourtant, si l'on accepte ce manque de pertinence il peut être intéressant d'indiquer les personnes qui font le plus souvent l'objet d'un article.

En inspectant le tableau $n^{\circ} 2$, on constate que de Gaulle l'emporte, et de loin, suivi de Proust et Camus ${ }^{25}$. Les noms de Gide et de Montherlant peuvent étonner, mais ils reflètent leur statut à cette époque après la guerre. 


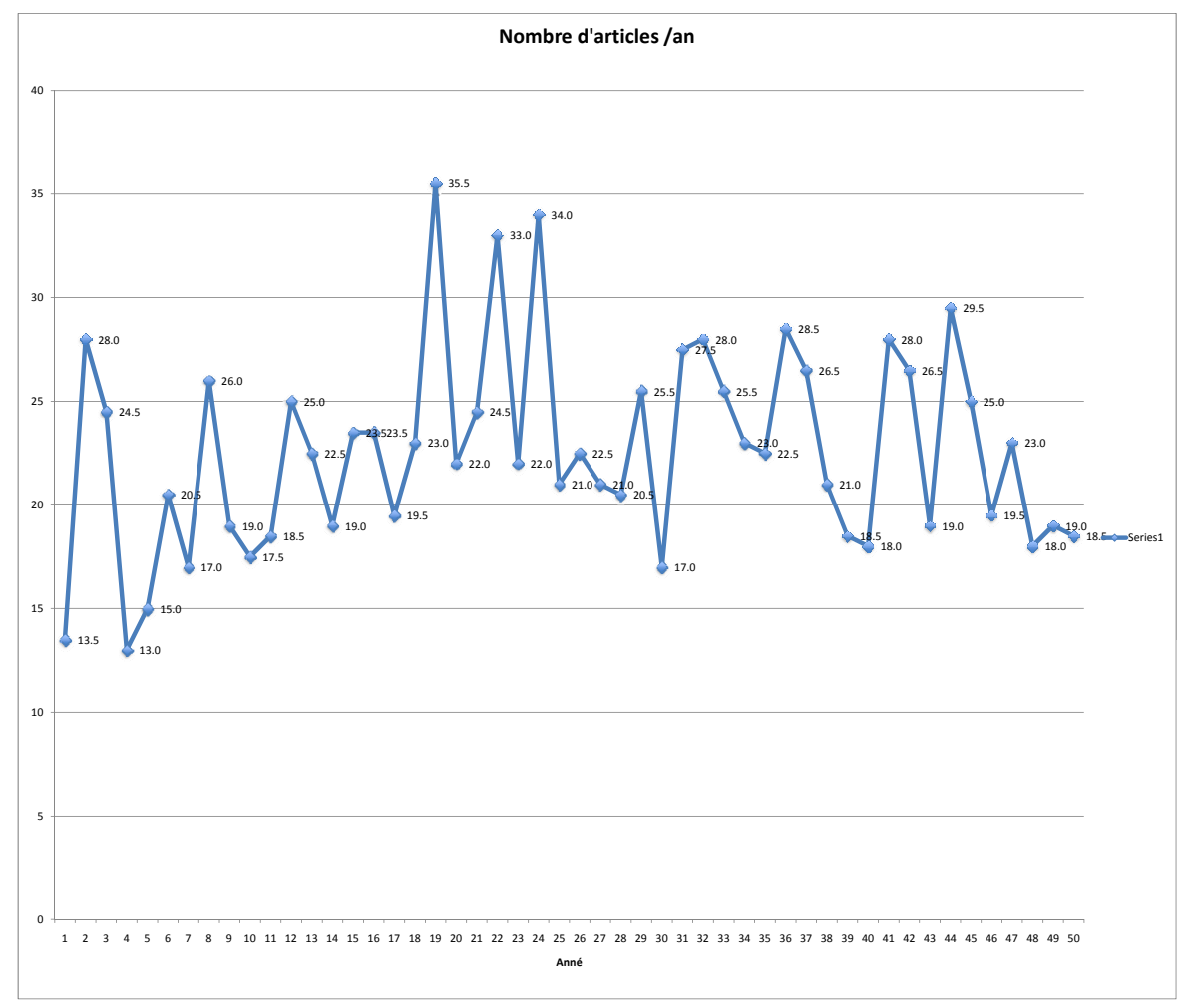

Graphique 1. Le nombre d'articles publiés Under strecket ayant comme sujet la France pendant les années I946-I995.

Le bon résultat obtenu par Balzac est dû au nombre d'articles qui lui sont consacrés dans la deuxième moitié de la période, tandis que l'intérêt pour Proust se maintient au même niveau à travers la période dans sa totalité.

On pourrait dire, pour résumer le contenu de ce tableau, que pendant la première moitié de la période ce sont des personnages contemporains qui tiennent le haut du pavé, tandis que des personnes déjà entrées dans l'Histoire retiennent l'attention pendant la deuxième période.

\section{Qui écrit les articles Under strecket ?}

Qui sont les auteurs de ces articles ? Deux groupes se distinguent : (a) ceux qui sont surtout des spécialistes de la France et de la culture française et qui écrivent peu d'articles sur d'autres sujets ; (b) ceux qui ont un éventail plus large et qui écrivent des articles sur plusieurs sujets, pas 


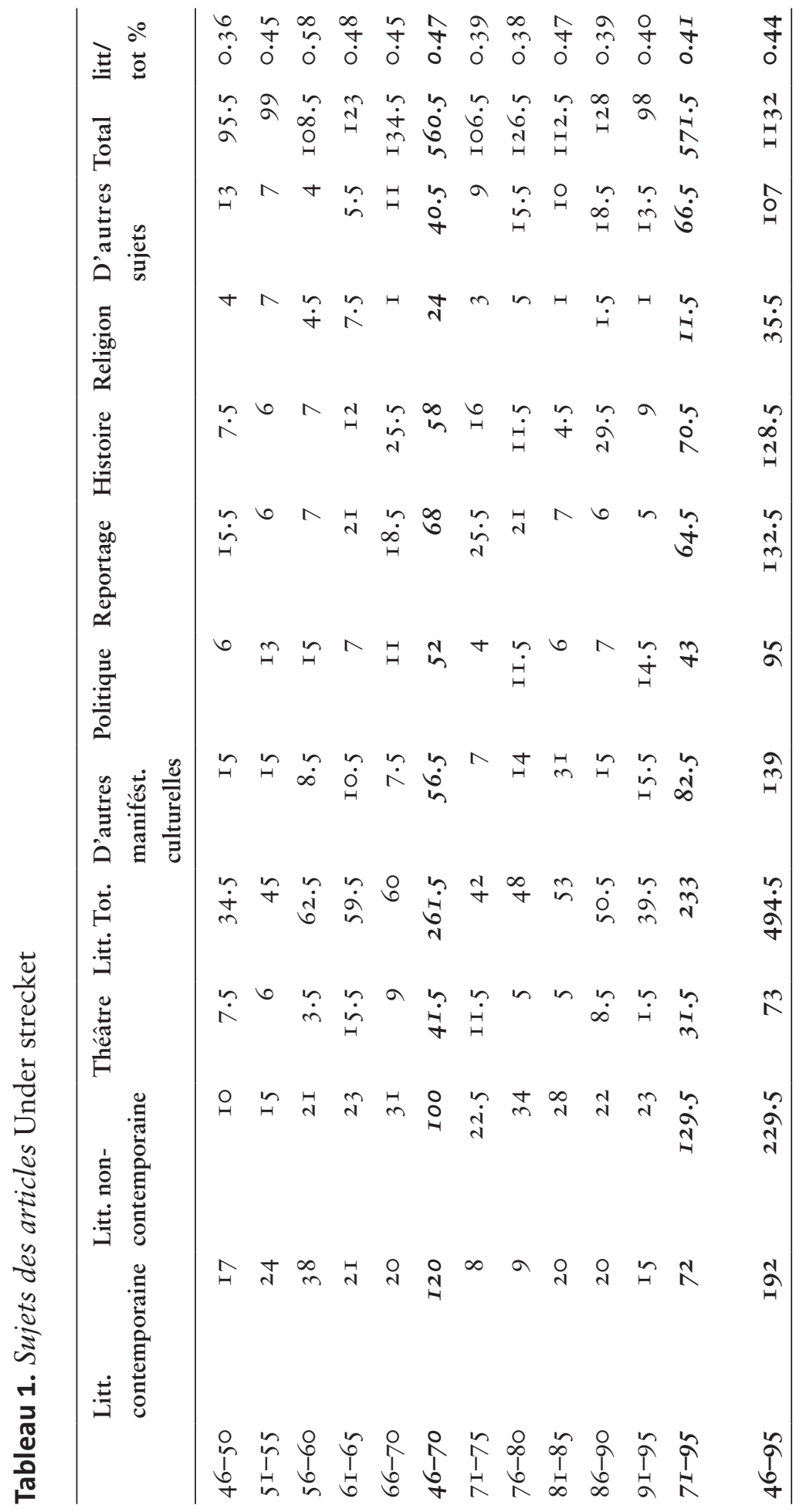




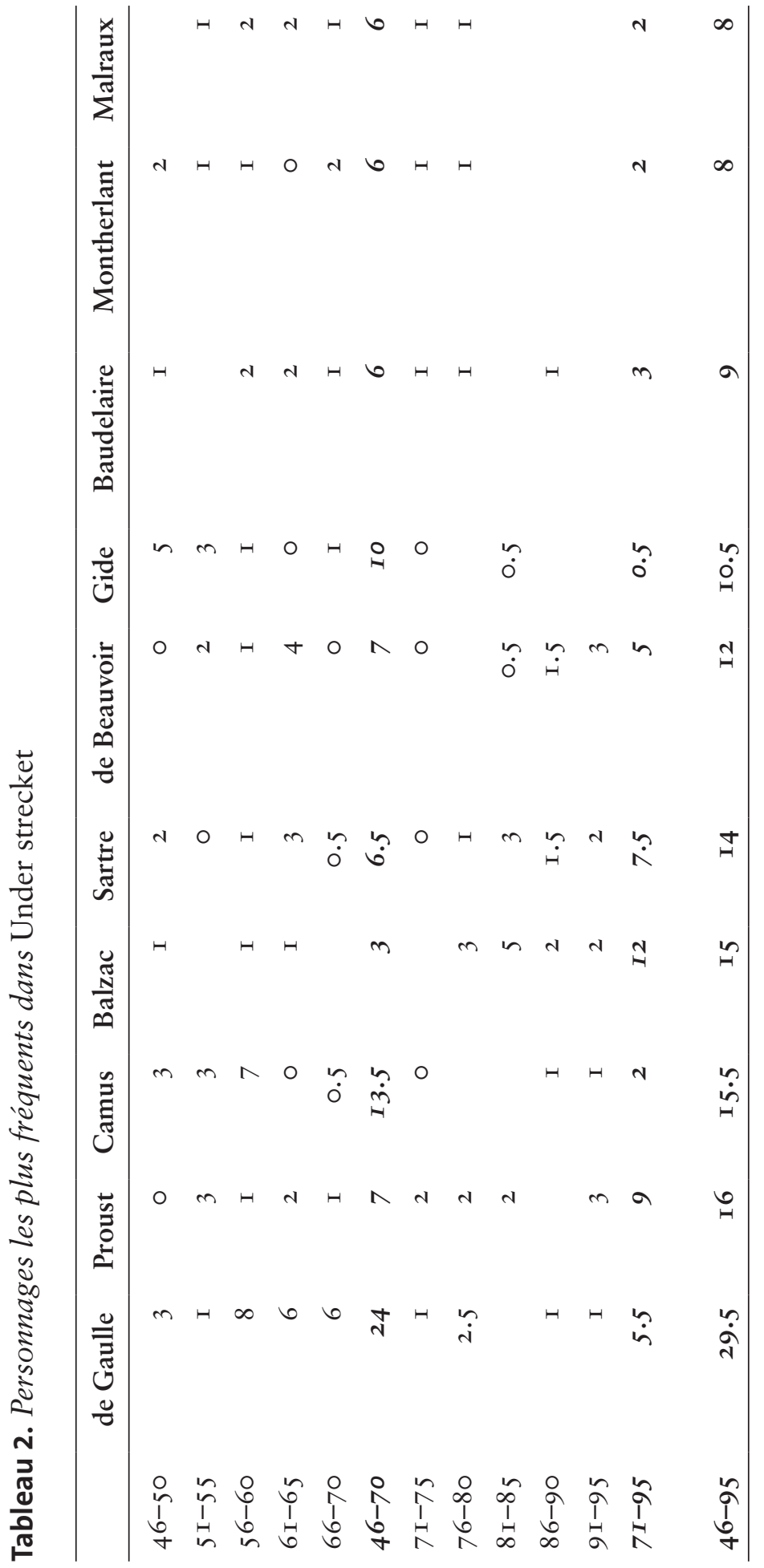




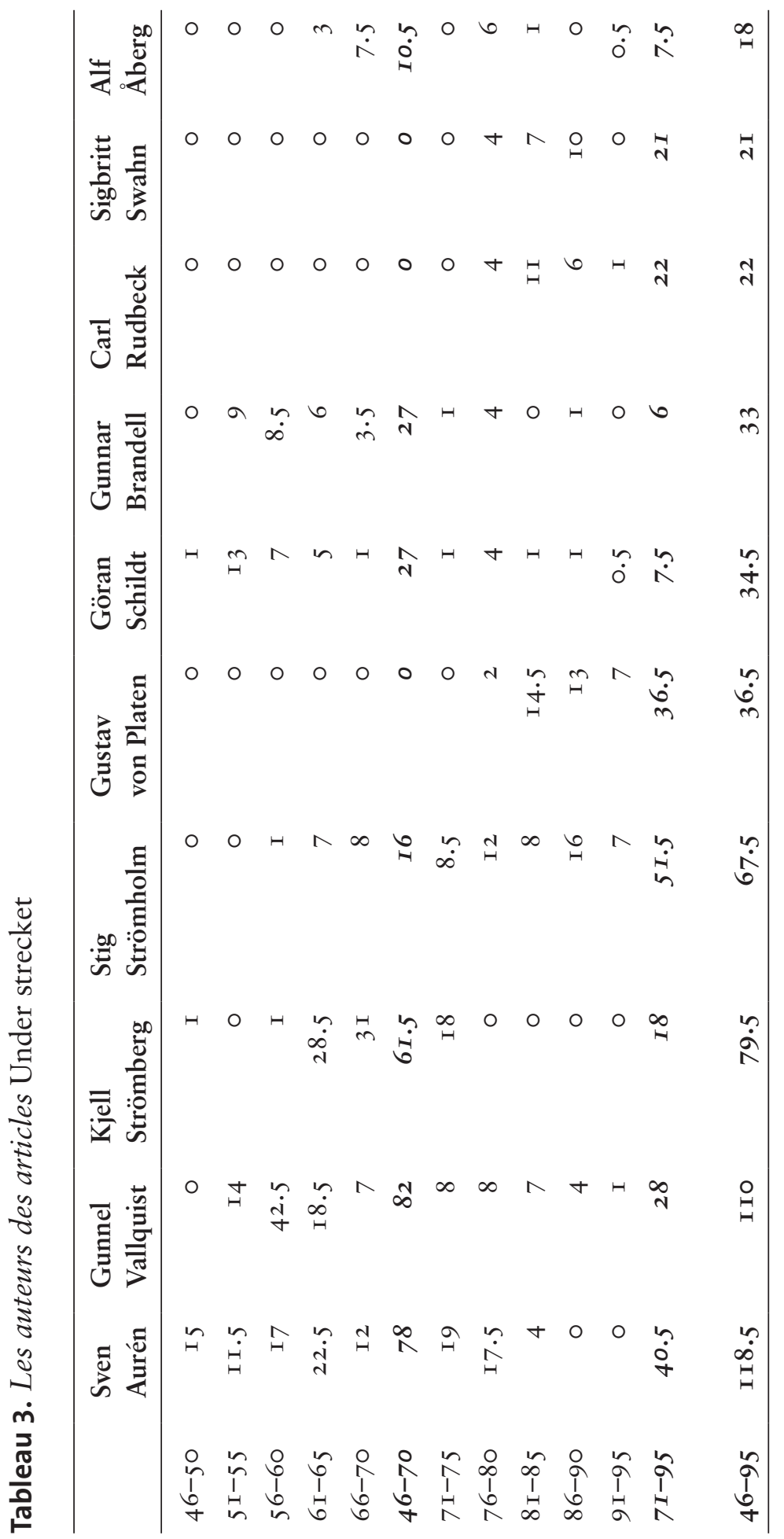


uniquement sur la France. Chaque groupe peut, à son tour, être divisé en deux : (c) les auteurs qui écrivent beaucoup et régulièrement des articles et (d) ceux qui écrivent peu d'articles.

Ce qui étonne, quand on regarde les noms des auteurs, c'est le nombre très restreint d'auteurs ayant écrit beaucoup d'articles. Il y a, en effet, une grande différence entre les quatre premiers auteurs, qui dominent, et les autres. Ces quatre contributeurs ont écrit entre I I 8 et 67 articles chacun soit au total un tiers de tous les articles sur la France publiés dans la rubrique Under strecket ${ }^{26}$. Sven Aurén (groupes a et c) fut le correspondant du Svenska Dagbladet à Paris entre 1945 et I985; Gunnel Vallquist (groupes b et c) est journaliste, critique, auteur, essayiste, traductrice et, depuis I982, membre de l'Académie suédoise ; Kjell Strömberg (groupes a et c) fut conseiller d'ambassade à Paris, il a surtout écrit des articles sur le théâtre à Paris dans Svenska Dagbladet; Stig Strömholm (groupes b et c) est auteur, professeur titulaire de droit, président de l'Université d'Uppsala.

\section{Comparaison avec d'autres pays}

Bien que le résultat, presque deux articles par mois Under strecket sur la France ou la culture française, soit tout à fait honorable et démontre que l'intérêt pour la France en Suède fut élevé pendant la période I946-I995, nous voulons toutefois le comparer avec d'autres articles Under strecket afin de pouvoir déterminer avec plus de précision l'importance de la culture française par rapport à d'autres pays dans la vie culturelle suédoise pendant cette période.

Publier un article sur la France est en même temps de la part de la rédaction une décision de ne pas publier un article sur un autre sujet. Jusqu'ici, dans notre enquête, nous n'avons que regardé de près le résultat pour la France, sans prendre en compte sa position relative vis à vis d'autres sujets ou pays. Le chiffre de I,9 article en moyenne par mois nous indique que la culture française est un sujet intéressant mais ne nous indique pas si elle l'est plus ou moins que d'autres sujets possibles.

Pour faire une telle comparaison, les autres pays qui s'imposent sont, bien entendu, l'Angleterre, les États-Unis, l'Italie, l'Allemagne, l'Autriche, les autres pays nordiques et la Russie/l'Union soviétique. Ces pays furent eux aussi importants dans la discussion culturelle en Suède après la deuxième guerre mondiale. Ils sont donc choisis parce qu'ils devraient être les pays qui, en premier lieu, entre en concurrence de l'attention pendant la période étudiée. Nous avons ajouté les articles 
qui traitent de l'Antiquité grecque et latine puisque l'on peut a priori supposer que ce sont des sujets intéressants pour les lecteurs de Svenska Dagbladet $^{27}$.

Ensemble, le nombre de ces articles s'élève à 6903 , représentant presque quarante pour cent de tous les articles Under strecket publiés entre I946 et I995. Le groupe augmente collectivement avec presque neuf pour cent entre la première et la deuxième moitié de la période, ce qui est un développement étonnant compte tenu du fait que le nombre d'articles consacrés au monde latino-américain, aux pays arabes et autres pays en dehors des pays occidentaux augmente aussi. Cette augmentation est surtout due à un intérêt croissant pour les États-Unis et l'Allemagne ${ }^{28}$, tandis que l'intérêt pour l'Angleterre et les pays nordiques recule beaucoup.

Globalement, on peut constater que les articles sur l'Angleterre sont les plus nombreux. Ce résultat n'est pas étonnant, étant donné le rôle prépondérant que ce pays a joué dans le monde occidental après la guerre - mais ce qui est plutôt surprenant, ce sont trois autres constatations.

D'abord, le nombre total d'articles consacrés à la France est inférieur à ceux consacrés à l'Angleterre, mais de peu. En effet dans les dernières vingt-cinq années de l'étude, le nombre dépasse même celui obtenu par l'Angleterre. À elle seule, la France est le deuxième pays pendant les dernières vingt-cinq années de la période, après les États-Unis, à attirer l'attention des auteurs qui écrivent Under strecket. Certes, l'Angleterre et les États-Unis obtiennent ensemble un score plus important, mais les auteurs de ces articles ne font jamais, ou presque, de lien entre les deux pays, et les sujets sont d'ailleurs trop spécialisés pour permettre un tel rapprochement. En outre, les articles sur la France représentent la moitié du nombre d'articles sur l'Angleterre et les États-Unis pris ensemble.

Deuxième constatation : il y a plus d'articles sur la France que sur l'Allemagne, un pays avec qui la Suède historiquement a eu des relations étroites.

Troisième constatation : la France et les pays nordiques font presque jeu égal pendant la première moitié de la période, ce qui assez remarquable compte tenu des liens très forts entre la Suède et ses voisins. Par contre (et encore plus étonnant), l'intérêt pour ces derniers s'affaiblit sensiblement pendant la deuxième moitié de la période de telle sorte que le nombre d'articles sur la France dépasse celui des pays nordiques de plus de vingt pour cent. 


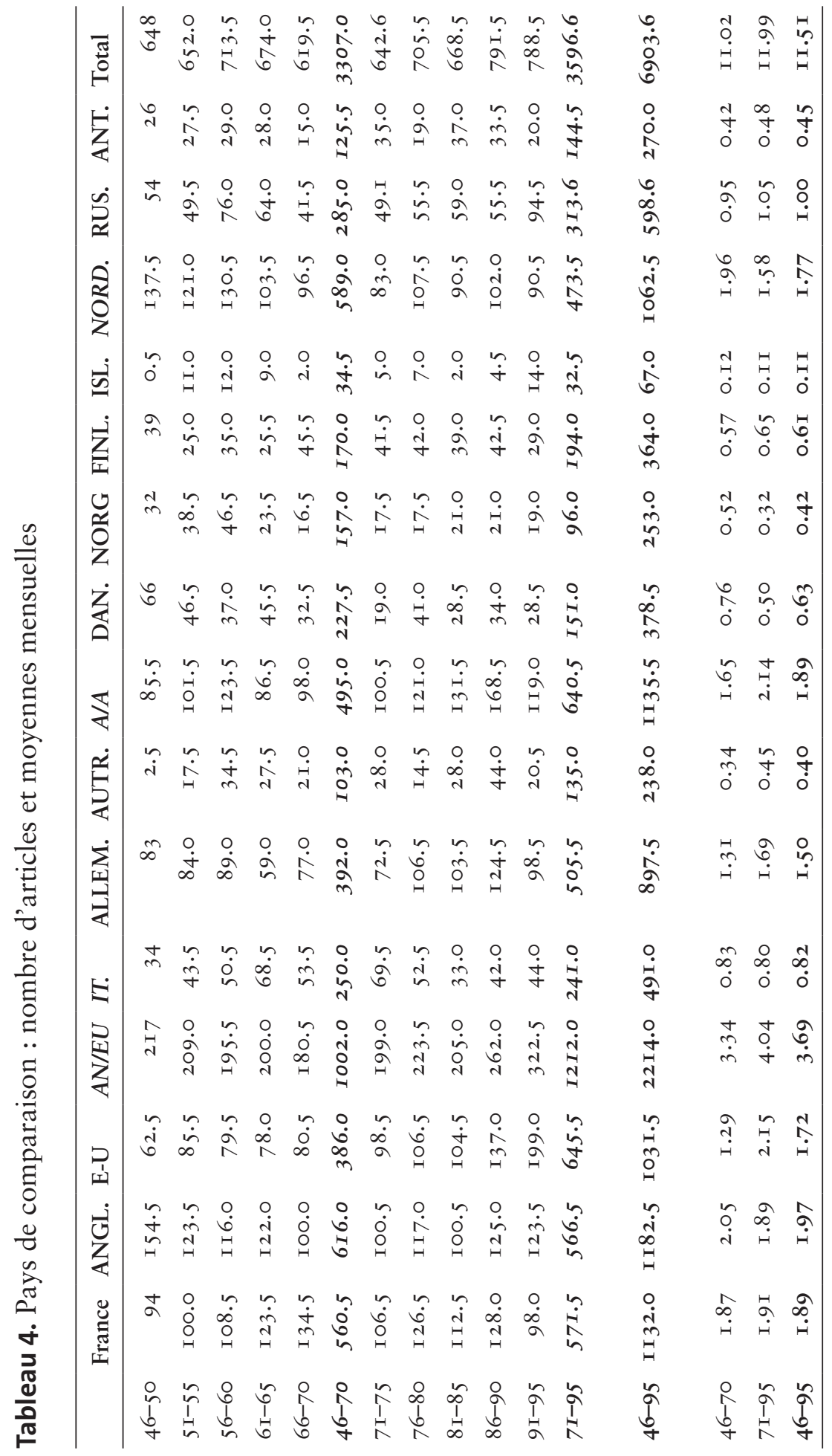




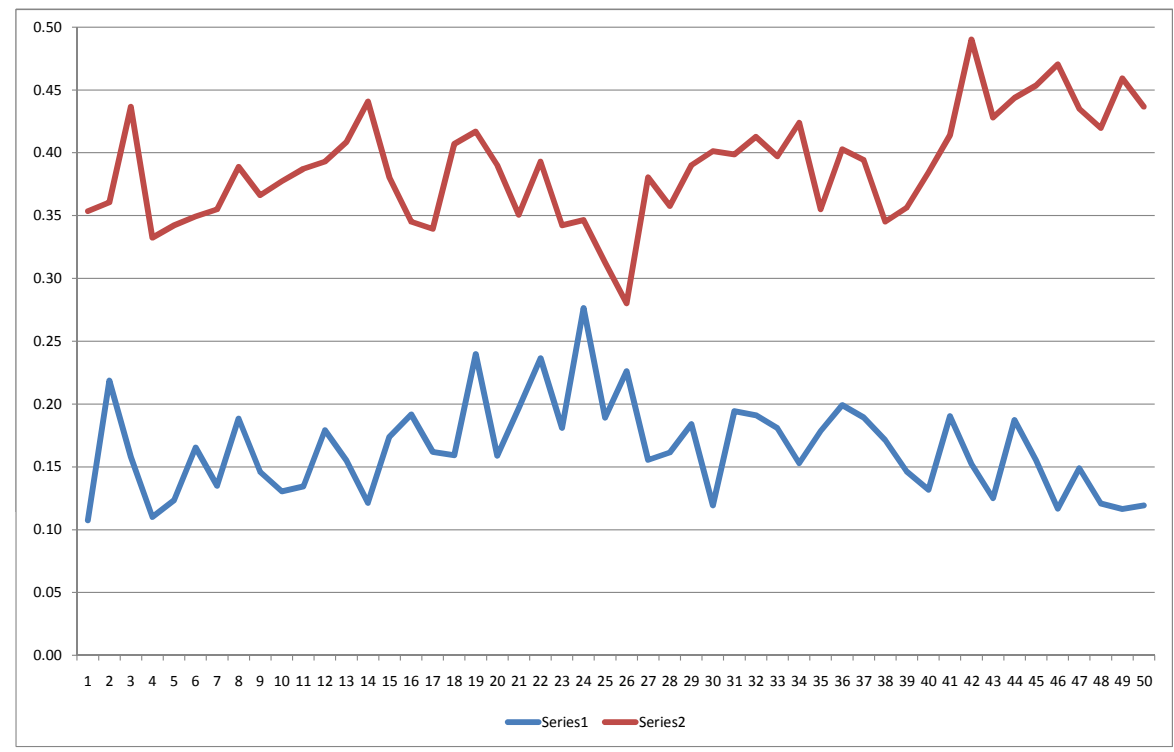

Graphique 2. Le nombre d'articles sur la France parmi le groupe «pays étrangers " (série I) et le nombre d'articles sur "les pays étrangers » parmi tous les articles publiés Under strecket (série 2).

Donc, ce tableau ne peut que renforcer notre conclusion selon laquelle qu'il y a eu en Suède, sur le plan culturel, un grand intérêt pour la France tout au long des cinquante années qui ont suivi la deuxième guerre mondiale.

La série $n^{\circ} \mathrm{I}$ dans le graphique $\mathrm{n}^{\circ}{ }_{2}$ montre que pendant les vingtcinq premières années, la part des articles sur la France parmi tous les articles dans le groupe "pays étrangers » augmente, tandis qu'une évolution inverse se produit pendant les vingt-cinq dernières années (une période où le nombre d'articles sur la France accroît légèrement).

La série $\mathrm{n}^{\circ}{ }_{2}$ illustre le fait que les articles sur "les pays étrangers » parmi tous les articles publiés Under strecket augmentent vers la fin de la période étudiée.

\section{Comment choisir un article Under strecket ? Le choix de la rédaction}

Les chiffres présentés ci-dessus reflètent les choix faits par la rédaction de la section culturelle dans Svenska Dagbladet (au moins à partir de la date où Under strecket est placé dans cette section ${ }^{29}$. 
On peut préalablement supposer que le choix de la rédaction reflète l'intérêt réel des lecteurs (ou du moins ce que la rédaction croit être l'intérêt des lecteurs pour tel ou tel sujet), puisque le journal, produit commercial, se vend sur un marché et doit satisfaire les attentes du lectorat. $\mathrm{Vu}$ dans cette perspective, il n'est pas étonnant que les articles sur l'Islande soient moins nombreux que ceux sur la France. L'hypothèse que l'on peut soutenir est, par conséquent, que les articles représentent grosso modo ce que les lecteurs apprécient de lire.

Au long de ces cinquante années que comprend l'enquête, on peut constater qu'il y a un facteur stable et quelques facteurs changeants.

Le facteur stable est le nombre d'articles sur la culture française qui, malgré quelques fluctuations, ne se développe pas dans une direction précise.

Les facteurs changeants sont (a) les auteurs et (b) le contenu des articles.

Même les auteurs les plus fréquents ne sont actifs qu'une partie de cette période, et la quantité de leurs contributions varie aussi d'une année à l'autre. Mais il est évident que la rédaction se fie à quelques auteurs dont les qualités sont connues. Dans un article du I 5 août I990, Ingmar Björkesten explique le pourquoi de Under strecket et insiste, en ce qui concerne les choix rédactionnels, sur les qualités stylistiques et intellectuelles des auteurs. Le côté essayiste ainsi que la possibilité, pour un auteur brillant, de faire vivre n'importe quel sujet sont des aspects particulièrement mis en avant. Les considérations commerciales, par contre, ne sont pas abordées ${ }^{30}$.

Quand, pendant la deuxième moitié de la période, le nombre d'articles sur la littérature contemporaine diminue alors que le nombre d'articles sur la littérature non-contemporaine augmente, l'explication semble être que la diminution entre les deux moitiés ne dépende pas d'un choix rédactionnel mais plutôt des préférences des auteurs. La rédaction veille sur la quantité d'articles sur tel ou tel pays ou sujet, mais se soucie moins du contenu ${ }^{3 \mathrm{I}}$. Cela expliquerait ainsi que les articles sur l'actualité des scènes parisiennes disparaissent presque complètement avec la mort de Kjell Strömberg. Par la suite en revanche, un certain nombre d'articles sur la peinture et des expositions d'art à Paris (des sujets moins suivis avant I980) sont écrits par Gustav von Platen, journaliste installé à Paris et ancien rédacteur en chef de Svenska Dagbladet. Les auteurs, une fois acceptés, semblent avoir la possibilité de se consacrer à leurs thèmes favoris. ${ }^{32}$ 
Toutes ces considérations nous amènent à conclure que : (a) la rédaction cherche à satisfaire la demande/l'intérêt des lecteurs ; (b) les auteurs proposent des articles qui, lorsqu'ils sont des auteurs « de la maison ", sont dans la grande majorité acceptés aussi longtemps que les " quotas » assignés à chaque pays ou chaque sujet ne dépassent pas la norme établie par la rédaction (une norme censée refléter l'intérêt réel des lecteurs) ; (c) la lecture de ces articles peut, à leur tour, changer la nature de la demande des lecteurs (et ainsi modifier " les quotas»).

\section{Notes}

I. Par exemple, le lien culturel entre la Suède (peu d'habitants) et l'Allemagne (beaucoup d'habitants) a historiquement été très fort, mais l'exportation de biens culturels vers la Suède l'emporte sur l'importation venue de la Suède.

2. Cf., par exemple, la différence entre un livre importé de France et un livre traduit du français en Suède.

3. Ce sont les termes qu'emploie Diana Cooper-Richet dans une analyse d'échanges culturels ( "Transferts culturels et passeurs de culture dans le monde du livre (France-Brésil), XIX siècle ", (20I3), Patrimônio e Mémória (vol. 9) I. Sâo Paulo : Unesp) ; les termes sont analysés, p. I3 I. L'introduction (transfert) en Suède de l'existentialisme français, ou du théâtre de Sartre, par Thure Stenström (passeur) dans des livres et articles (canaux) en sont des exemples.

4. Il faut aussi prendre en compte les voies indirectes par lesquelles des influences pénètrent dans une autre culture (l'existentialisme dans sa version française peut, par exemple, être importé de l'Allemagne après avoir fait le détour par des philosophes allemands).

5. L'intérêt suédois pour la France remonte au Moyen Âge où « nous rencontrons des individus et des groupes qui recherchaient un échange culturel entre [la Suède et] la France ", Östman, M. \& H. (2008), Au Champ d'Apollon; Écrits d'expression française produits en Suède (1 550-2006). Filologiskt Arkiv 47 . Kungl. Vitterhets Historie och Antikvitets Akademien : Motala : 9. Voir aussi Battail, M. \& J.-F. (I993), Une amitié millénaire. Les relations entre la France et la Suède à travers les âges. Paris : Kungl. Vitterhets Historie och Antikvitets Akademien.

6. Östman (2008 : 42) note que dans « les salons de l'époque de Bernadotte [roi de Suède et ancien maréchal de Bonaparte], le français avait [...] un rang important, comparable à celui qu'il avait atteint pendant l'ère gustavienne [...]. »

7. Dans ce contexte, il ne faut pas oublier l'importance de la mode et de la 
cuisine françaises, toujours très présentes (sous forme d'articles ou de publicité) dans les quotidiens et les journaux, surtout pendant les années quarante et cinquante.

8. Les produits commerciaux (on peut penser aux voitures françaises) emportent avec eux aussi une part de la culture parce qu'ils sont perçus comme des produits fort ancrés dans la culture française.

9. Après la guerre, les influences anglo-saxonnes dans les domaines cinématographiques, littéraires et musicales furent profondes dans tous les pays occidentaux. Il faut aussi prendre en considération les liens traditionnellement très forts entre la Suède et l'Allemagne et les relations proches entre la Suède et les autres pays nordiques, forgées pendant des siècles et fondées sur une culture partagée, des langues semblables (en dehors du finnois) et une histoire commune. Dans cette perspective, le rôle de la France ne peut qu'être relativisé.

Io. Certains reportages dans Svenska Dagbladet, surtout les week-ends, peuvent, en effet, parfois occuper encore plus d'espace (avec des illustrations) mais ils contiennent rarement plus de signes.

I I. Svenska Dagbladet est un quotidien fondé en I 884 et au format tabloïde. Under strecket existe comme partie à part depuis I9I9. Avant I965, Under strecket était placé à des pages différentes, comme à l'éditorial. La critique de livres et de spectacles (pas toujours dans le même endroit dans le quotidien) n’a pas été régulière pendant la période étudiée. Après mars I990, l'article, toujours au bas de la page, continue sur l'autre page (une page paire).

I 2. Cela ne signifie pas que tous les intellectuels lisent Svenska Dagbladet, seulement que Svenska Dagbladet couvre en profondeur les domaines culturels et intellectuels d'une manière à satisfaire les exigences de ce lectorat.

I 3. Il faut ici souligner, avant de continuer notre enquête, que les articles publiés Under strecket ne représentent qu'une partie de tous les articles publiés et que beaucoup d'articles sur la France et la culture française sont publiés ailleurs dans Svenska Dagbladet.

I4. À cause d'une grève, Svenska Dagbladet ne fut pas publié entre le 2 et le 9 juin 1989 .

I 5. Exclus sont donc d'autres pays francophones (le Canada, la Belgique, la Suisse, etc.).

I6. Les seules exceptions sont Simenon, Beckett et Ionesco (pour ces deux derniers quand leurs ouvrages sont écrits en français et connectés à la culture française).

I7. Un article ne fait pas partie du corpus s'il contient une discussion sur un phénomène français, ou un personnage français, qui occupe moins de cin- 
quante pour cent de l'espace (exemple : le I 7 janvier I955 et un article sur le monologue intérieur chez Joyce, Wagner et Valéry).

I 8. Sauf, pendant la première moitié de la période, les articles deviennent de plus en plus fréquents.

I9. La distribution des articles tout au long de l'année est aléatoire : il se peut que plus de trois mois s'écoulent sans un article sur la France et, d'un autre côté, trois articles peuvent être publiés trois jours de suite (cas extrême : 20-22 octobre I 986 - sur Simone de Beauvoir, l’abbé Prévost et Luc Ferry).

20. Le groupe "Littérature contemporaine " comporte des auteurs vivants, ou récemment décédés. Sartre et de Beauvoir appartiennent à ce groupe. «Reportage » peut, par exemple, être des impressions faites lors d'un voyage dans une ville ou région de France ou un article sur la mentalité et l'esprit français. Dans le groupe «D'autres manifestations culturelles », on trouve surtout la peinture. Le groupe «D'autres sujets » inclut la philosophie et les philosophes.

2 I. La raison pour cette diminution peut être la participation plus faible de Gunnel Vallquist dont les articles ont souvent eu un thème actuel.

22. L'augmentation pour la littérature non-contemporaine entre la première et la deuxième moitié de la période s'explique en partie par les contributions de Stig Strömholm dont bon nombre d'articles portent sur la littérature française entre I600-I945. Il faut aussi mentionner les articles de Sigbrit Swahn pendant cette deuxième moitié, surtout sur Balzac.

23. En effet, pour la période I975-I995, uniquement trois articles portent sur ce sujet.

24. Comme nous avons indiqué ci-dessus, Under strecket fonctionne uniquement comme un baromètre, cet endroit dans le journal ne reflète pas, l'intérêt global de tel ou tel personnage parce qu'ils sont aussi (dans beaucoup de cas) mentionnés et commentés dans d'autres sections dans le journal, comme la critique.

25. Le chiffre pour de Gaulle $(29,5)$ peut être comparé avec ceux pour Churchill (36) et Wagner (34).

26. Les quinze auteurs les plus fréquents représentent 53 pour cent des articles.

27. Le monde espagnole, latino-américain, arabe et chinois ainsi que les pays en voie de développement sont rarement d'actualité dans les années cinquante et soixante et ne figurent pas, pour cette raison, parmi nos pays de référence. Suivant les mêmes critères qui dictent la définition de la culture française, "L'Angleterre », par exemple, n'inclut pas d'autres pays anglo-saxons (comme l'Irlande, le Canada et l'Australie). 
28. À partir des années soixante-dix, le domaine «culturel " s'élargit et incorpore de plus en plus de manifestations culturelles auparavant exclues d'une discussion "sérieuse "-comme la musique pop et le jazz. C'est surtout les articles sur les États-Unis qui en profitent. Ce changement ne semble pas avoir laissé de traces dans les articles sur la France. L'augmentation pour l'Allemagne s'explique en partie par la nouvelle situation en Europe après I989, mais l'augmentation commença dix ans plus tôt.

29. La section culturelle dans Svenska Dagbladet a une rédaction et son propre rédacteur en chef (la section en a eu cinq pendant la période étudiée). Ce sont alors les rédactions et leurs rédacteurs en chef successifs en collaboration avec les auteurs qui sont les passeurs ou, selon le terme employé par Pierre Bourdieu (2002) - dans l'article « Les Conditions sociales de la circulation internationale des idées". Actes de la recherche en science sociales I45:5-des sélectionneurs.

30. Bourdieu (2002:4), nous rappelle que publier un article n'est pas un acte gratuit - au contraire, c'est faire de la publicité pour soi-même et « renforcer [sa] position dans le champ [culturel] ». Parmi les cinq auteurs les plus assidus, trois d'entre eux furent des journalistes à Svenska Dagbladet, pour les deux autres (Strömberg et Strömholm), il est difficile de s'exprimer mais on peut ici oser l'observation que ni pour un diplomate ni pour un académicien, publier des articles dans un quotidien n'est pas un mérite très important. Au moins dans le cas de Strömholm (un " détenteur de positions académiques dominantes ", ibid.), nous pouvons avec un peu de certitude soutenir l'hypothèse que ses contributions sont faites peut-être surtout dans la volonté de sensibiliser le lecteur pour des valeurs " classiques".

3 I. Dans un monde de plus en plus international, la France a toujours un rôle à jouer, tandis que l'espacement des articles sur les autres pays nordiques témoigne d'une relation moins intime et moins ressentie et, donc, dans l'ensemble moins intéressante pour les lecteurs (dans les années quarante, un Under strecket pouvait même être écrit en norvégien ou en danois, chose impensable dix ans après). La question du contenu est épineuse. Gunnel Vallquist a écrit plusieurs articles sur des auteurs catholiques et on peut se demander si ce fait reflète une stratégie rédactionnelle ou non (plutôt non). Pourtant, la rédaction ne se désintéresse jamais complètement du contenu, les nombreux articles sur la révolution française (lors du bicentenaire) et sur l'évolution sociale et économique en Russie après I989 en sont des preuves.

32. Notre propre expérience (basée sur une contribution de plus de quarante articles Under strecket après la période étudiée ici) corrobore cette constatation. La question que pose Bourdieu (ibid.), "Pourquoi est-ce untel qui a publié untel ? ", est importante, mais il n'est pas possible d'y répondre en restant dans les limites de notre enquête. 


\section{Bibliographie}

Battail, M. \& J.-F. (dir.) (I993), Une amitié millénaire. Les relations entre la France et la Suède à travers les âges. Stockholm : Kungl. Vitterhets Historie och Antikvitets Akademien.

Bourdieu, P. (I989), "Les conditions sociales de la circulation internationale des idées ». Conférence à l'université de Fribourg le 30 octobre I989. <http://www.espacesse.org/bourdieu-I.php>

Cooper-Richet, D. (2OI3), "Transferts culturels et passeurs de culture dans le monde du livre (France-Brésil, XIX siècle) », Patrimônio e Mémória, vol. 9: I, I 28-I 43 .

Svenska Dagbladet, 6.903 articles «Under strecket » entre I946 et I995.

Östman, M. \& Hans (2008), Au Champ d'Apollon. Écrits d'expression française produits en Suède (I 550-2006). Motala : Filologiskt Arkiv 47 ; Kungl. Vitterhets Historie och Antikvitets Akademien. 